\title{
DESENVOLVIMENTO DE UMA UNIDADE EXPERIMENTAL PARA ESTUDOS EM TERMOSSIFÕES COM APLICAÇÃ̃O DA TÉCNICA DE PIV PARA ANÁLISE DO ESCOAMENTO
}

\author{
J. WEISE $^{1 *}$, R. K. DECKER ${ }^{1}$, D. NORILER ${ }^{1}$, E. OLIVEIRA ${ }^{2}$, H. F. MEIER ${ }^{1}$ \\ ${ }^{1}$ Universidade Regional de Blumenau, Departamento de Engenharia Química \\ ${ }^{2}$ Petróleo Brasileiro S. A. (PETROBRAS), Abastecimento, Tecnologia de Refino, Utilidades \\ *e-mail: josiane.weise@gmail.com
}

\begin{abstract}
RESUMO
O escoamento induzido pelas forças de empuxo em um termossifão é avaliado no presente estudo, com ênfase na consolidação de uma bancada experimental para constituição de base empírica, a ser empregada na formulação e validação de modelos matemáticos de CFD para este tipo de sistema. O termossifão da unidade experimental compreende um downcomer e um riser, que consistem em tubos de vidro enjaquetados, e um separador líquido-vapor. Como medida experimental, o perfil de velocidade axial na região do riser foi obtido por meio da técnica de velocimetria por imagem de partícula (PIV), para três condições operacionais do regime monofásico e do bifásico, variando-se a temperatura de entrada da água nas jaquetas do downcomer e do riser. Para o regime monofásico, o perfil de velocidade axial é caracterizado por velocidades mais altas na região próxima à parede em todas as condições avaliadas, seguindo o perfil esperado para a temperatura. Em regime bifásico, com o aumento do número de bolhas formadas pela ebulição do líquido, o perfil de velocidade axial torna-se mais uniforme ao longo do raio e a magnitude da velocidade aumenta significativamente, em comparação aos resultados obtidos para o regime monofásico.
\end{abstract}

\section{INTRODUÇÃO}

Em sistemas do tipo termossifão, ou circuitos de circulação natural, o escoamento é estabelecido pela força de empuxo que se desenvolve devido à presença de gradientes de densidade. Em regime monofásico, estes gradientes são induzidos por diferenças de temperatura, enquanto em regime bifásico, taxas de circulação maiores podem ser atingidas em função da vaporização do fluido, a qual resulta em uma diferença significativa entre a densidade do líquido e da mistura líquido-vapor, presentes nos ramais verticais do sistema.

A simplicidade construtiva, o baixo consumo interno de energia e a operação passiva dos termossifões, justificam sua variedade de aplicações, seja em geradores de vapor, em refervedores de colunas de destilação, em sistemas de aquecimento de água por energia solar, ou em sistemas de refrigeração, que incluem desde o controle térmico de componentes eletrônicos até o resfriamento de reatores nucleares.

A fenomenologia envolvida nos sistemas do tipo termossifão tem sido extensivamente investigada, tanto por experimentação física quanto numérica. Por meio de um aparato experimental composto por um evaporador e um condensador conectados por dois tubos, Khodabandeh e Furberg (2010), investigaram a influência de diferentes geometrias para o evaporador na transferência de calor, no 
regime de escoamento e nas instabilidades do sistema termossifão bifásico. Franco e Filippeschi (2013), projetaram um termossifão bifásico fechado, de pequenas dimensões e conduziram testes experimentais, a fim de avaliar a relação entre a taxa mássica através do sistema e o fluxo de calor imposto. Basu et al. (2013), investigaram a hidrodinâmica de um termossifão através de um aparato experimental composto por dois risers e um downcomer conectados a um separador de fases. Ar e água sob condições ambientes foram utilizados como fluidos de teste, de modo que o ar comprimido foi injetado a uma velocidade conhecida na corrente líquida, através de aspersores montados na parte inferior de cada riser. Para caracterizar o escoamento bifásico, os autores utilizaram sondas de condutividade e técnicas fotográficas.

Quanto aos estudos numéricos, em regime monofásico, Pilkwal et al. (2007), Angelo et al. (2012) e Wang, Chang e Ferng (2013), aplicaram técnicas de CFD para simulação de sistemas de circulação natural, obtendo boa concordância entre resultados físicos e numéricos. No entanto, para o caso dos sistemas bifásicos, a convecção natural que ocorre simultaneamente à mudança de fase, torna a modelagem destes fenômenos complexa, uma vez que um modelo satisfatório deve incorporar termos associados à ebulição e à transferência de calor e quantidade de movimento entre as fases, bem como os mecanismos de transferência de calor a partir da parede (GANDHI et al., 2011).

Diante das vantagens que a simulação numérica introduz na análise de anomalias operacionais, estudos de melhoria de desempenho e otimização geométrica e operacional, o objetivo deste estudo consiste na consolidação de um aparato experimental para constituição de base empírica, que sustente a formulação e a validação de modelos matemáticos de CFD para termossifões bifásicos. Com este intuito, a avaliação preliminar da unidade é realizada mediante a análise dos perfis de velocidade axial no riser, obtidos por meio da técnica de PIV, em diferentes condições operacionais, tanto em regime monofásico quanto bifásico.

\section{MATERIAIS E MÉTODOS}

\subsection{Descrição da Unidade Experimental}

A unidade experimental compreende o termossifão propriamente dito e os sistemas de aquecimento/ resfriamento e condensação associados, como mostra a Figura 1.

O termossifão é composto por um tubo descendente (downcomer), um tubo ascendente (riser) e o separador líquido-vapor. Tanto o riser quanto o downcomer correspondem a trocadores de calor duplo tubo, fabricados em vidro, cuja altura total é igual a $1.340 \mathrm{~mm}$ e a região de troca térmica apresenta $1.000 \mathrm{~mm}$ de altura. Os tubos internos possuem diâmetro externo de $52 \mathrm{~mm}$ e espessura de parede de $2,5 \mathrm{~mm}$, enquanto as jaquetas apresentam diâmetro externo de $105 \mathrm{~mm}$ e espessura de parede de $3 \mathrm{~mm}$.

Acetona é empregada como fluido de trabalho, devido ao seu baixo ponto de ebulição $\left(56^{\circ} \mathrm{C}\right)$. No downcomer $(1)$, a acetona é resfriada, enquanto no riser (2) ocorre o seu aquecimento e a mudança de fase. A circulação é estabelecida devido a diferença entre a densidade do líquido no downcomer e a densidade do líquido, ou da mistura líquidovapor, no riser. Mangueiras, com terminais em aço carbono, fazem a conexão entre o downcomer e o riser, bem como entre o riser e o separador líquido-vapor (3), o qual é fabricado em aço inoxidável e consiste em um reservatório cilíndrico de $10 \mathrm{~L}$, com diâmetro de $200 \mathrm{~mm}$ e comprimento de $300 \mathrm{~mm}$.

O sistema de aquecimento/ resfriamento, que fornece a água que circula na região anular do downcomer e do riser, é composto por dois banhos ultratermostáticos. O banho ultratermostático (4) utilizado para retirar energia do líquido que circula no downcomer 
tem capacidade de $9 \mathrm{~L}$ e vazão de $7 \mathrm{~L} / \mathrm{min}$. Já para o fornecimento de energia ao riser, o banho ultratermostático (5) empregado tem capacidade de $11 \mathrm{~L}$ e a vazão da bomba de circulação externa é de $10 \mathrm{~L} / \mathrm{min}$.

A condensação do vapor de acetona é realizada por um trocador de calor duplo tubo (6), fabricado em aço inoxidável. O trocador é posicionado verticalmente, de modo que o condensado retorne para o separador líquidovapor. A água que circula na jaqueta do condensador é proveniente de um banho termostático (7) com vazão de $4 \mathrm{~L} / \mathrm{min}$.
Entradas para medidas de temperatura e pressão estão disponíveis anteriormente e posteriormente à região de troca térmica do downcomer e do riser e no separador líquidovapor, onde também ocorre a aquisição de medidas de pressão. Os sensores de temperatura são do tipo PT-100 (8), e a pressão é medida através de um transmissor de pressão (9), calibrado para a faixa de 0 a 1 bar.

\subsection{Velocimetria por Imagem de Partícula}

A técnica de Velocimetria por Imagem de Partícula, conhecida pela sigla PIV (do

Figura 1 - Representação tridimensional da unidade experimental

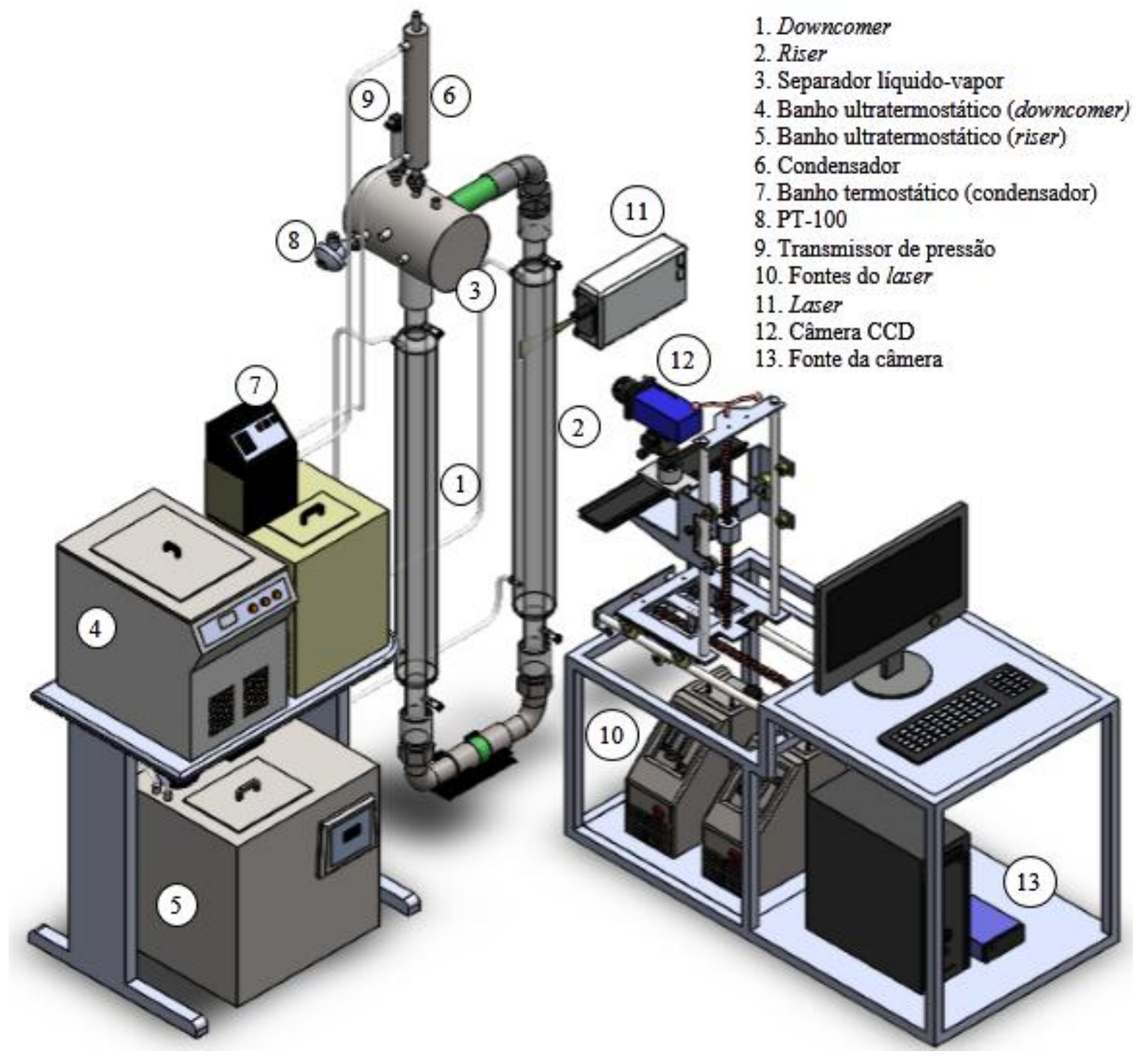


inglês Particle Image Velocimetry), foi desenvolvida com o objetivo de mapear instantaneamente os campos de velocidade em um escoamento. A grande vantagem introduzida pela técnica consiste na possibilidade de se obter informações sobre a velocidade do escoamento em todo o plano de análise, e com alta resolução. Todos os métodos conhecidos até então forneciam apenas valores pontuais para a velocidade (RAFFEL, WILLERT e KOMPENHANS, 1998). A PIV apresenta também a vantagem de ser uma técnica não intrusiva, pois não se faz necessária a imersão de sondas, as quais podem causar perturbações ao escoamento, dificultando sua análise.

Para a utilização da técnica de PIV, fazse necessária a adição de partículas traçadoras ao escoamento. No arranjo mais convencional, que corresponde ao PIV-2D, um pulso plano de laser ilumina a região a ser analisada. A luz refletida pelas partículas traçadoras é captada por uma câmera do tipo CCD (do inglês Charged Coupled Device), que produz a primeira imagem. Após um curto intervalo de tempo, o laser é disparado novamente, e a segunda imagem é produzida.

Para determinação do campo de velocidades, as imagens produzidas são divididas em pequenas subáreas denominadas janelas de interrogação. $\mathrm{O}$ vetor deslocamento das partículas traçadoras é então determinado para cada janela de interrogação por meio de métodos estatísticos de correlação cruzada, assumindo que as partículas dentro de uma mesma janela se movem de forma homogênea entre os dois pulsos de laser. $\mathrm{O}$ vetor velocidade é então calculado com base no intervalo de tempo. $\mathrm{O}$ processo é repetido para todas as janelas de interrogação do par de imagens, resultando no completo diagrama vetorial do escoamento em estudo.

O sistema PIV empregado no presente estudo, apresentado na Figura 1, compreende duas fontes de laser Nd:YAG (10) de alta potência modelo Ultra, da Quantel, com sistema ótico de duas cabeças (11), e uma câmera CCD (12) de alta resolução, modelo Imager Pro Plus/Pro X 2M (1600 x 1200 pixels) com fonte modelo VC-Imager Pro X, da La Vision (13). Um filtro com ponto de corte em $550 \mathrm{~nm}$ foi posicionado em frente à câmera, o qual bloqueia a reflexão do laser em comprimentos de onda inferiores a $550 \mathrm{~nm}$ e transmite-a em comprimentos de onda mais elevados.

Para aquisição das imagens, partículas traçadoras de dióxido de titânio, com diâmetro médio de Sauter de $1 \mu \mathrm{m}$ foram adicionadas à acetona. A câmera CCD foi posicionada perpendicularmente ao feixe de laser, sendo a base da área de medição $(47$ x $47 \mathrm{~mm})$ posicionada a $725 \mathrm{~mm}$ do início da região de troca térmica do riser. $\mathrm{O}$ tempo entre os dois pulos do laser foi estabelecido como 0,01 s. A aquisição e o pós-processamento das imagens foram realizados por meio do software DAVIS 8.1 da LaVision.

\subsection{Procedimento Experimental}

A metodologia para a execução dos experimentos é sintetizada em quatro etapas principais: (a) preenche-se o sistema termossifão com acetona; (b) ajusta-se a temperatura dos banhos termostáticos; (c) adicionam-se as partículas traçadoras e calibrase o sistema de PIV; (d) após a estabilização da temperatura da acetona, procede-se com a aquisição das imagens.

\section{RESULTADOS E DISCUSSÃO}

No sistema em estudo, as variáveis controláveis correspondem às temperaturas do banhos que promovem a circulação da água nas jaquetas do downcomer e do riser, enquanto que, como variáveis de resposta, foram mensurados a temperatura da acetona, adquirida na conexão entre o separador líquido-vapor e o downcomer, e o perfil de velocidade axial no riser, obtido através da técnica de PIV, na altura de $740 \mathrm{~mm}$ da região 
de troca térmica do riser.

\subsection{Regime Monofásico}

Os experimentos em regime monofásico foram realizados em duplicata, variando-se a temperatura de entrada da água na jaqueta do riser em $50{ }^{\circ} \mathrm{C}, 60^{\circ} \mathrm{C}$ e $70^{\circ} \mathrm{C}$, e adotando-se uma temperatura inferior em $15^{\circ} \mathrm{C}$ para a entrada da água na jaqueta do downcomer.

A temperatura observada para a acetona na entrada do downcomer, nos dois experimentos realizados, é apresentada na Tabela 1, em conjunto com a respectiva condição operacional. Verifica-se que a temperatura de estabilização da acetona, para uma mesma condição operacional, apresentouse em concordância nos dois experimentos.

$\mathrm{Na}$ Tabela 1, verifica-se ainda que entre as condições operacionais M1 e M2, um incremento de aproximadamente $8{ }^{\circ} \mathrm{C}$ é observado na temperatura da acetona. Já entre as condições M2 e M3, a temperatura do sistema eleva-se em aproximadamente $6{ }^{\circ} \mathrm{C}$, uma vez que a acetona atinge uma temperatura ligeiramente superior à temperatura de ebulição, mas não muda de fase. Este comportamento do sistema está associado à característica do fenômeno de ebulição nucleada de que o crescimento das bolhas ocorre em pontos discretos da superfície aquecida, os quais são denominados sítios de

Tabela 1 - Regime Monofásico: Condições operacionais e temperatura da acetona.

\begin{tabular}{ccccc}
\hline Exp. & $\begin{array}{c}\text { Condição } \\
\text { Operacional }\end{array}$ & $\begin{array}{c}\text { Temperatura da água } \\
\text { na entrada da jaqueta } \\
\text { do downcomer }\left({ }^{\circ} \mathrm{C}\right)\end{array}$ & $\begin{array}{c}\text { Temperatura da água } \\
\text { na entrada da jaqueta } \\
\text { do } \text { riser }\left({ }^{\circ} \mathrm{C}\right)\end{array}$ & $\begin{array}{c}\text { Temperatura da acetona na } \\
\text { entrada da região de troca } \\
\text { térmica do downcomer }\left({ }^{\circ} \mathrm{C}\right)\end{array}$ \\
\hline 1 & M1 & 35 & 50 & 41,8 \\
1 & M2 & 45 & 60 & 50,3 \\
1 & M3 & 55 & 70 & 56,4 \\
2 & M1 & 35 & 50 & 42,1 \\
2 & M2 & 45 & 60 & 50,2 \\
2 & M3 & 55 & 70 & 56,4 \\
\hline
\end{tabular}

Figura 2 - Perfis radiais de velocidade axial média $\left(\overline{\mathrm{v}}_{\mathrm{z}}\right)$ obtidos no regime monofásico de operação

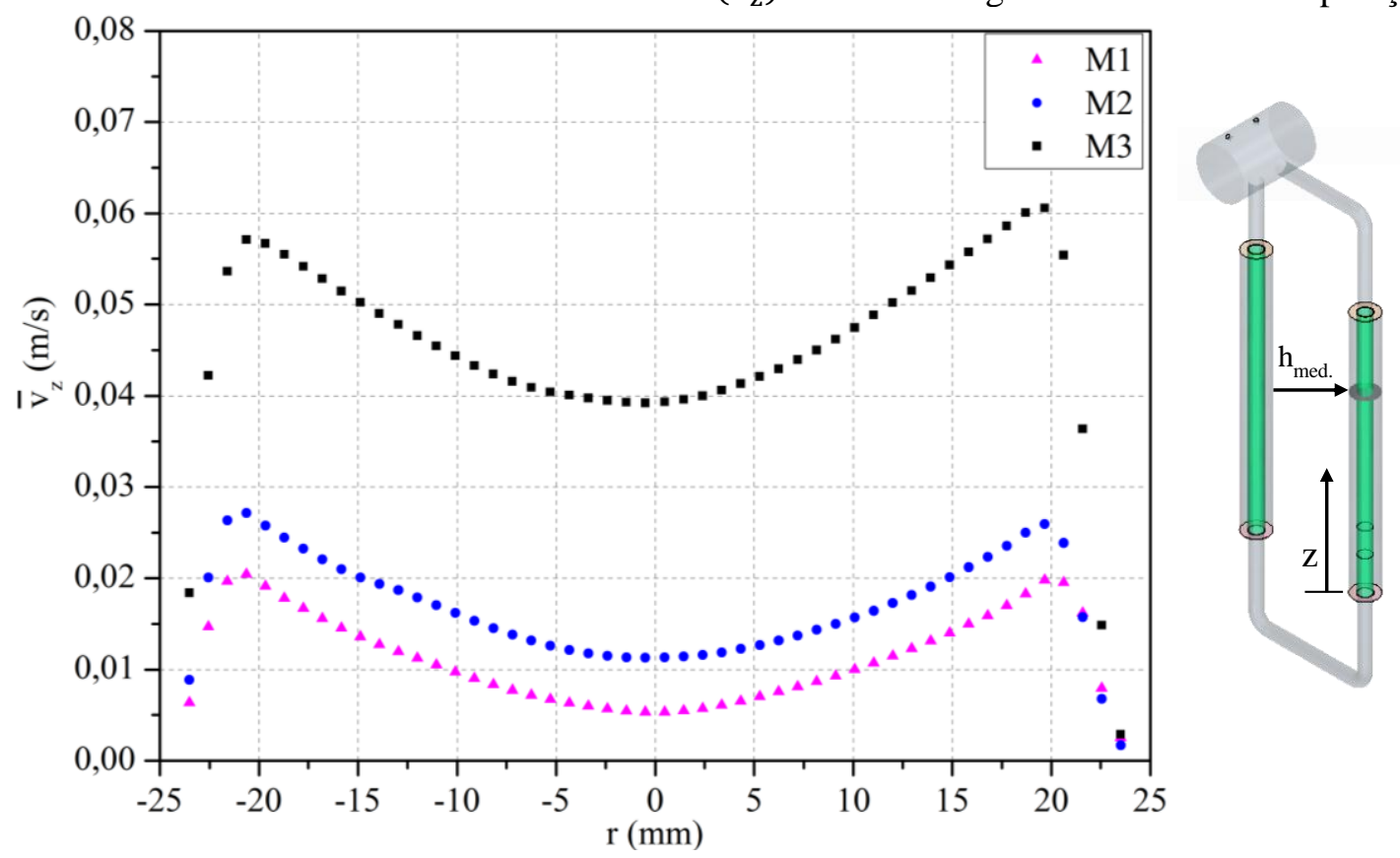


nucleação. Em geral, com o aumento da temperatura e da rugosidade da superfície, o número de sítios também aumenta. No entanto, superfícies muito lisas, tal como a do vidro, podem levar ao superaquecimento do líquido e provocam um atraso no processo de ebulição.

$O$ perfil de velocidade, em cada experimento e para cada condição operacional, foi calculado a partir da média dos perfis instantâneos obtidos no processamento de 1.500 pares de imagens.

A Figura 2 apresenta a média dos perfis de velocidade axial dos dois experimentos, nas três condições operacionais analisadas, para os quais observa-se um comportamento semelhante: a velocidade é maior na região próxima à parede e diminui em direção ao centro do tubo, o que está de acordo como o perfil de temperatura esperado. $\mathrm{O}$ comportamento característico é resultante das forças de empuxo, que induzem à ascensão vertical do líquido na região próxima à parede, devido ao seu aquecimento. O líquido que ascende, por sua vez, arrasta o fluido das camadas adjacentes, além de aquecê-lo. O movimento observado é oscilatório, embora esta informação não seja traduzida pelos perfis médios de velocidade.

Comparando-se os perfis de velocidade axial apresentados na Figura 2, verifica-se que, apesar da diferença de temperatura entre a água na região de entrada da jaqueta do downcomer e do riser ser constante, o acréscimo na temperatura da parede do riser, aumenta a velocidade do escoamento, especialmente na condição M3. O aumento da temperatura modifica as propriedades do líquido, o que, consequentemente, altera o balanço entre a força de empuxo, que induz o movimento, e as forças viscosas.

Uma maior diferença é observada na magnitude dos perfis de velocidade da condição M2 para a condição M3, frente à variação da condição $\mathrm{M} 1$ para a $\mathrm{M} 2$, embora $\mathrm{o}$ incremento de temperatura da acetona tenha se apresentado menor. Esta alteração mais evidente está relacionada ao fato de que as propriedades do fluido não apresentam uma variação linear com a temperatura. Assim, para um gradiente fixo de temperatura, a variação das propriedades do líquido em temperaturas mais elevadas é superior à variação apresentada em temperaturas mais baixas.

\subsection{Regime Bifásico}

Com base em uma série de experimentos, desenvolvidos em condições operacionais diversas, constatou-se que a superfície do vidro é incapaz de promover a nucleação das bolhas na região do riser, conforme discutido na seção 3.1. Assim, para permitir a análise do sistema em regime bifásico, uma região de superfície mais rugosa que o vidro foi criada, a partir da introdução de um anel de alumínio no interior do riser, de modo a garantir as condições necessárias para o desenvolvimento do fenômeno de ebulição. $\mathrm{O}$ anel foi confeccionado a partir de uma chapa de alumínio jateada, com $100 \mathrm{~mm}$ de altura e $0,8 \mathrm{~mm}$ de espessura, e foi posicionado na altura de $100 \mathrm{~mm}$ do início da região de troca térmica do riser.

As temperaturas de $60^{\circ} \mathrm{C}, 65^{\circ} \mathrm{C}$ e $70{ }^{\circ} \mathrm{C}$, na jaqueta do riser, foram combinadas às temperaturas de $45^{\circ} \mathrm{C}, 55^{\circ} \mathrm{C}$ e $55^{\circ} \mathrm{C}$ na jaqueta do downcomer, respectivamente, para a avaliação do comportamento do sistema em regime bifásico. As condições operacionais avaliadas e a temperatura observada para a acetona na entrada do downcomer, são apresentadas na Tabela 2, enquanto os perfis de velocidade axial média do líquido são expostos na Figura 3. O perfil de velocidade axial média para a condição B1 foi obtido a partir da aquisição e processamento de 2.000 imagens. Já nas condições B2 e B3, 6.000 imagens foram utilizadas.

A partir dos dados apresentados na Tabela 2, observa-se que, após a introdução do anel de alumínio, a nucleação das bolhas sobre o anel ocorre mesmo que a temperatura da acetona encontre-se abaixo da temperatura de 
saturação $\left(56{ }^{\circ} \mathrm{C}\right)$.

De acordo com a Figura 3, as condições B1 e B2 para o regime bifásico apresentam um comportamento muito semelhante ao observado para o regime monofásico, em que o aumento da temperatura nas proximidades da parede altera a densidade do fluido, de modo que as forças de empuxo promovem à ascensão vertical mais intensa do líquido nesta região. $\mathrm{Na}$ condição B2, no entanto, o pico de velocidade ocorre em uma distância maior em relação à parede frente à condição B1. Já para a condição B3, não é mais possível identificar um pico na velocidade axial ao longo do perfil. Em ambas as condições, B2 e B3, a presença das bolhas altera o campo de velocidade do líquido, tornando o perfil de velocidade axial mais uniforme na região central do tubo.
Verifica-se ainda que, em comparação ao regime monofásico, a velocidade axial do líquido é significativamente superior no regime bifásico, com exceção da condição B1. Nesta condição, o perfil de velocidade bifásico mostrou-se ligeiramente inferior ao monofásico, frente às mesmas condições impostas para as temperaturas de entrada da água nas jaquetas. Visualmente, o número de bolhas apresentou-se muito pequeno na condição B1, e assim, não influenciou positivamente na razão de circulação.

\section{CONCLUSÔES}

Neste estudo, um sistema termossifão experimental foi desenvolvido e o escoamento em regime monofásico e bifásico foi avaliado

Tabela 2 - Regime Bifásico: Condições operacionais e temperatura da acetona.

\begin{tabular}{cccc}
\hline $\begin{array}{c}\text { Condição } \\
\text { Operacional }\end{array}$ & $\begin{array}{c}\text { Temperatura da água na } \\
\text { entrada da jaqueta do } \\
\text { downcomer }\left({ }^{\circ} \mathrm{C}\right)\end{array}$ & $\begin{array}{c}\text { Temperatura da água na } \\
\text { entrada da jaqueta do } \\
\text { riser }\left({ }^{\circ} \mathrm{C}\right)\end{array}$ & $\begin{array}{c}\text { Temperatura da acetona na } \\
\text { entrada da região de troca } \\
\text { térmica do downcomer }\left({ }^{\circ} \mathrm{C}\right)\end{array}$ \\
\hline $\mathrm{B} 1$ & 45 & 60 & 50,3 \\
$\mathrm{~B} 2$ & 55 & 65 & 55,9 \\
$\mathrm{~B} 3$ & 55 & 70 & 56,1 \\
\hline
\end{tabular}

Figura 3 - Perfis radiais de velocidade axial média $\left(\bar{v}_{z}\right)$ obtidos no regime bifásico de operação

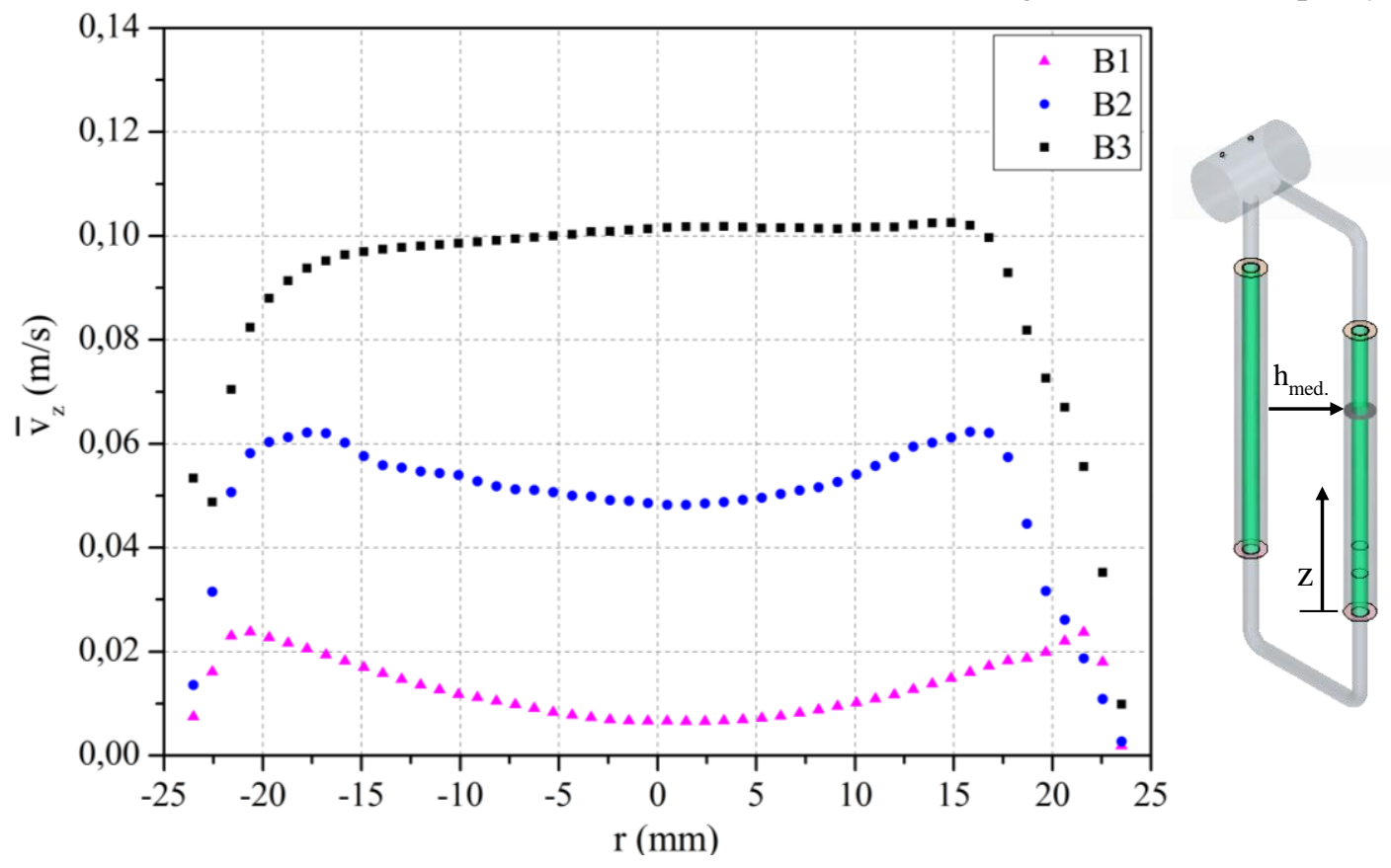


por meio dos perfis de velocidade axial obtidos pela técnica de PIV.

Para o regime monofásico de operação, identificou-se um perfil de velocidade axial característico em que a velocidade é maior nas proximidades da parede e decresce na direção do centro do tubo, conforme esperado para os gradientes de temperatura e, consequentemente, para os gradientes de densidade no interior do termossifão. Identificou-se também que o aumento da temperatura do fluido que circula no termossifão produz um incremento significativo na magnitude da velocidade.

Para o regime bifásico, observou-se que com o aumento da quantidade de bolhas, $o$ perfil de velocidade axial é alterado, deixando de apresentar picos nas regiões próximas da parede, para formar um perfil de velocidade mais uniforme ao longo do raio.

\section{REFERÊNCIAS BIBLIOGRÁFICAS}

ANGELO, G.; ANDRADE, D. A.; ANGELO, E.; TORRES, W. M.; SABUNDJIAN, G.; MACEDO, L. A.; SILVA, A.F. A numerical and three-dimensional analysis of steady state rectangular natural circulation loop. Nuclear Engineering and Design, v. 244, p. 61-72, 2012.

BASU, D. N.; PATIL, N. D.; BHATTACHARYYA, S.; DAS, P. K. Hydrodynamics of a natural circulation loop in a scaled-down steam drum-riser-downcomer assembly. Nuclear Engineering and Design, v. 265 , p. 411-423, 2013.

FRANCO, A.; FILIPPESCHI, S. Experimental analysis of Closed Loop Two Phase Thermosyphon (CLTPT) for energy systems. Experimental Thermal and Fluid Science, v. 51, p. 302-311, 2013.

GANDHI, M. S.; SATHE, M. J.; JOSHI, J. B.; VIJAYAN, P. K. Two phase natural convection: CFD simulations and PIV measurement. Chemical Engineering Science, V. 66, P. 3152-3171, 2011.

KHODABANDEH, R.; FURBERG, R Instability, heat transfer and flow regime in a two-phase flow thermosyphon loop at different diameter evaporator channel. Applied Thermal Engineering, v. 30, p. 1107-1114, 2010.

PILKHWAL, D. S.; AMBROSINI, W.; FORGIONE, N.; VIJAYAN, P. K.; SAHA, D.; FERRERI, J. D. Analysis of the unstable behaviour of a single-phase natural circulation loop with one-dimensional and computational fluid-dynamic models. Annals of Nuclear Energy, v. 34, p. 339-355, 2007.

RAFFEL, M.; WILLERT, C. E.; KOMPENHANS, J. Particle image velocimetry: a practical guide. New York: Springer, v. XVI, 1998. 253 p.

WANG, J. Y.; CHUANG, T. J.; FERNG, Y. $M$. CFD investigating flow and heat transfer characteristics in a natural circulation loop. Annals of Nuclear Energy, v. 58, p. 65-71, 2013.

\section{AGRADECIMENTOS}

Os autores agradecem ao suporte financeiro da PETROBRAS, através do termo de cooperação 0050.0070334.11.9, e ao CNPQ - Processo 310504/2012-0, que tornaram possível a realização deste trabalho. 